\title{
Diversity and Distribution of Invasive Plant Species in Suburban Vegetation of Kashmir Himalayas
}

\author{
Hamayun Shaheen*, Ammara Batool, Syeda Fatima Gillani, \\ Muhammad Ejaz ul Islam Dar, Tariq Habib, Shamshad Aziz \\ Department of Botany, University of Azad Jammu and Kashmir, Muzaffarabad, Pakistan
}

Received: 1 May 2018

Accepted: 24 June 2018

\begin{abstract}
Biological invasion has been considered one of the most important mechanisms of global change, posing serious threats to the conservation of native ecosystems world-wide. The present study was conducted in the sub-tropical vegetation stands in the suburban areas of Muzaffarabad city to study the diversity and distribution of invasive alien species (IAS) of plants and assess the factors responsible for their spread. A total of 9 sites, including 2 in the city and 7 in suburban areas, were sampled using random quadrate methods. The sampled flora were classified according to the phytogeographic origin and invasion status, whereas the phytosocioogoical attributes of plant communites were also calculated following standard protocols. A total of 121 plant species represented by 108 genera belonging to 58 families was recorded from the study area. Results revealed that $35.6 \%$ flora comprised of 43 species were recorded as IAS. The IAS dominating the area included Parthenium hysterophorus, Lantana camara, Xanthium strumarium, Ailanthus altissima, Cannabis sativa, Broussonetia papyrifera, Arundo donax and Sorghum halepense. Analysis of the Invasion status revealed that invasive aliens had a weight of $46 \%$ followed by naturalized aliens and casual aliens, respectively. The dominance of invasive species resulted in decreased species diversity at the disturbed sites. Landslides and road construction were identified as factors promoting the establishment and spread of invasive species in the area. Principal component analysis revealed significant correlation of edaphic disturbances with the abundance of IAS. An integrated management system is recommended for early detection and control of IAS.
\end{abstract}

Keywords: biological invasion, diversity, suburban vegetation, land slides, road construction

*e-mail: hamayunmaldial@yahoo.com 


\section{Introduction}

Biological invasion has been considered an important mechanism of global change posing serious threats to the conservation of native ecosystems worldwide [1]. The invasion of alien plant species in the new regimes has become the second highest threat to plant diversity after habitat loss [2,3]. Under the Convention on Biological Diversity, invasive alien species (IAS) means species, subspecies or lower taxon, introduced outside its natural past or present distribution; includes any part, eggs, gametes, propagules or seeds of such species that might stay alive and subsequently reproduce; and whose introduction and spread threaten biological diversity. High competitive ability, reproductive effectiveness, efficient dispersal, vegetative reproduction, rapid organization and other traits help IAS adapt to new habitats $[4,5]$.

The impacts of IAS are globaly significant, long-term and commonly classified as economic, environmental or social in nature. IAS can impede species diversity, richness, composition, abundance and interactions, including mutualisms. The direct effects of IAS at the species level occur through processes such as predation, competition with and parasite transmission to individual organisms $[6,7]$. IAS can lead to the fragmentation, destruction, alteration and even the whole replacement of habitats, often resulting in consequences for even more species and ecosystem processes, primary to the functional collapse of the native ecosystem [8]. The potential risk of invasion is directly correlated with human activities synchronized with environmental disturbances and stresses. The disturbed and secondary vegetation is vulnerable to the violently invading invasive alien species $[9,10]$.

The western Himalayan biotic province is reported to have about 700 alien plant species [11]. The state of Azad Jammu and Kashmir (AJK) is istuated in the Pir Panjal Mountain Range in the western Himalayas, having diverse phytoclimatic zones and harboring high levels of biological diversity [12]. Due to rapid socioeconomic transitions synchronized with population rise, defiorestation and urbanization, the local vegetation is highly exposed to the risks of invasions by alien floral elements, potentially leading to environmental degradation and loss of biodiversity [13, 14]. Despite the intensity of the threat, there is no available data about the invasive alien flora in the area. The curent study was designed to investigate the diversity and distribution of invasive species in the suburban areas of Muzaffarabad, the capital of AJK. Specific objectives included analyzing the status of the invasive species along with inestigating the factors responsible for the spread of invasive species in suburban vegetation.

\section{Experimental Procedure}

\section{Study Area}

Muzaffarabad is the capital of the state of Azad Jammu and Kashmir situated between $34.24^{\circ}$ latitude and $73.22^{\circ}$ longitude in northeastern Pakistan spreading over an area of $1642 \mathrm{~km}^{2}$. The topography of Muzaffarabad is mountainous and hilly, characterized by subtropical scrub and pine forests [15]. The climate

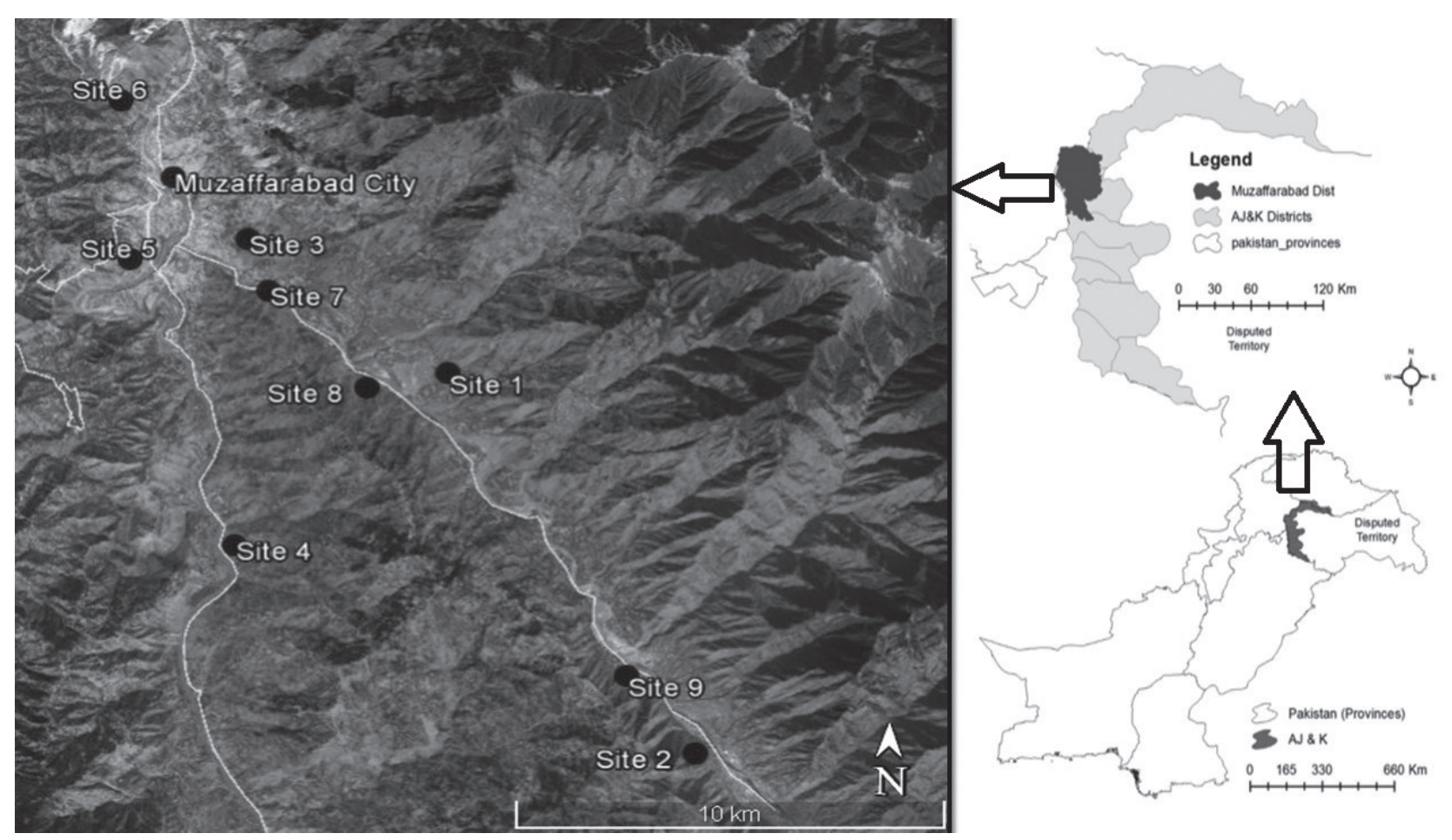

Fig 1. Map of the study area and satellite imagery of the sampling sites. 
of the area falls under subtropical and humid type with considerable variations. June and July are the hottest months, with maximum temperature a 40.0 to $47.5^{\circ} \mathrm{C}$ and minimum as 28.0 to $37.5^{\circ} \mathrm{C}$, respectively, while January and February are the coldest months with maximum temperatures of $15.0-21.5^{\circ} \mathrm{C}$ and minimum $-3.0--1.0^{\circ} \mathrm{C}$. The average annual precipitation of the district is $1511 \mathrm{~mm}$, with maximum rainfall during monsoon and early spring season [16].

\section{Methodology}

A total of 9 sites were selected for recording the vegataion composition and community structure having 2 sites in the city area, and 7 in the suburban areas (Fig. 1). The data was recorded systematically using the quadrats method with $1010 \times 10 \mathrm{~m}$ quadrats for trees at each site. Each quadrat was subdivided into $5 \times 5 \mathrm{~m}$ sample plots for recording shrubs and samplings, and $1 \mathrm{x} 1 \mathrm{~m}$ for herbs and seedlings. The herbaceous cover was determined by Daubenmire's cover scale. Trees with diameter at breast height $(1.5 \mathrm{~m})$ were measured to obtain basal area [17]. The density, frequency and coverage/basal areas was converted to relative values and added to obtain importance values index (IVI). The phytosociological attributes of the studied vegetation were determined, including species diversity, evenness, leaf spectrum, life form, richness and maturity indexes [18]. The plants were submitted to the herbarium of the Department of Botany, University of AJK Muzaffarabad, and were identified with the help of flora and available literature. The flora was evaluated and categorized on the basis of invasiveness, endemism, history and mode of introduction with the help of published literature and e-floras. The phytogeography analysis of the flora was performed to classify the floral aemements on the basis of continental origion [19]. The invasive alien species were further classified into Invasive, casual, naturalized, casual naturalized and cultivated classes [20]. Results were statistically analyzed by PCA to extract the significant correlations from species data set. The statistical analysis was performed by using software package PAST version 3.20.

\section{Results}

\section{Floristic Composition and Biological Spectra}

A total of 121 plant species belonging to 58 families and 101 genera were recorded from the study area. The major contribution of local flora included Asteraceae 11 species with a weight age of $10 \%$ followed by Poaceae 10 species with a weight age of $8 \%$. Rosaceae and Lamiaceae had 6 species with a weight age of $5 \%$, and Euphorbiaceae were represented by 5 species with a weight age of $4 \%$. Amaranthaceae, Cyperaceae, Papilionaceae, Pteridaceae and Verbenaceae had 4 species with a weight age of $3 \%$. Brassicaceae,
Polygonacaea and Moraceae were represented by 3 species with a weight age of $2 \%$, whereas Meliaceae, Apocynaceae, Oleaceae, Malvaceae, Convolvulaceae, Solanaceae, Plantaginaceae, Salicaceae and Anacardiaceae were represented by 2 species with a weight age of $2 \%$ each. The remaining 36 families had single representative with a weight age of $30 \%$. Herbs dominated the alien flora and were represented by 65 species, followed by shrubs ( 28 species), trees (21 species) and climbers (3 species), whereas 4 ferns were also recorded from the area. Therophytes were the dominant life form represented by 31 species with a weight age of $25.61 \%$. Nanophanerophytes were represented by 28 species with a weight age of $23.14 \%$. Megaphanerophytes life form was represented by 22 species $(18.18 \%)$, followed by Hemicryptophytes with 19 species $(15.71 \%)$. Geophytes were represented by 18 species (14.87\%); Lianas were represented by 3 species $(2.47 \%)$, whereas Chemophytes were represented by a single species. The dominant leaf size spectrum was microphyll, represented by 60 species with a weight age of $49.5 \%$. Leptophylls were represented by 22 species (18.18\%), followed by Nanophylls (21 species) and Mesophylls (18 species), respectively (Table 1).

\section{Phytosociological Attributes}

Phytosociological investigations revealed that the average species count at the sites was 43.7 with a maximum of 52 at site 5, whereas a minimum of 16 were found at sites 7 and 9 each. The study sites showed uniform values of Simpson's and Shannon-Wienner's diversity, averaging as 0.96 and 2.6, respectively attributed to the abundance of the common subtropical indicator species. The values of species richness showed significant variation ranging from 1.2 to 2.51 and correspeonding to the differential species count at different sites. The suburban vegetation communities revealed a very low value of maturity index with an average of mere $32.7 \%$, and none of them qualifying as mature (Table 2).

\section{Invasive Alien Flora}

A total of 43 invasive alien plant species belonging to 25 families and 41 genera were recorded from the area. The dominnat IAS included Parthenium hysterophorus with an IVI of 11.77, followed by Lantana camara (10.59), Xanthium strumarium (9.92), Ailanthus altissima (8.59), Cannabis sativa (7.78), Broussonetia papyrifera (7.28), Arundo donax (6.05) and Sorghum halepense (3.11). The major families that contributed to the alien flora included Asteraceae, Poaceae, Amaranthaceae, Brassicaceae, Euphorbiaceae, Fabaceae, Moraceae, Polygonaceae, Plantaginaceae and Salicaceae. The results of IAS classification on the basis of invasion status revealed that invasive aliens were represented by 20 species with a weight of $46 \%$, followed by naturalized aliens represented by 11 species (26\%). 
Table 1. Species composition, biological spectra and importance values of the suburban flora.

\begin{tabular}{|c|c|c|c|c|c|c|}
\hline $\mathrm{S} / \mathrm{no}$ & Botanical name & Family & Habit & Life form & Leaf spectra & IVI \\
\hline 1 & Acacia modesta Wall. & Mimosaceae & Tree & $\mathrm{Mp}$ & $\mathrm{Me}$ & 3.178 \\
\hline 2 & Achyranthes aspera L. & Amaranthaceae & Herb & $\mathrm{H}$ & $\mathrm{Mi}$ & 0.74 \\
\hline 4 & Adiantum caudatum L. & Pteridaceae & Fern & G & $\mathrm{N}$ & 0.36 \\
\hline 3 & Adiantum venustum D. Don. & Pteridaceae & Fern & G & $\mathrm{N}$ & 0.72 \\
\hline 6 & Ailanthus altissima (Mill) Swingle & Simaroubaceae & Tree & $\mathrm{Mp}$ & $\mathrm{Me}$ & 8.59 \\
\hline 5 & Ajuga bracteosa Var. alba (Gurke) Engl. & Lamiaceae & Herb & $\mathrm{Th}$ & $\mathrm{Mi}$ & 2.03 \\
\hline 7 & Alternanthera pungens Kunth. & Amaranthaceae & Herb & $\mathrm{H}$ & $\mathrm{N}$ & 1.33 \\
\hline 8 & Amaranthus spinosus L. & Amaranthaceae & Herb & Th & $\mathrm{N}$ & 3.61 \\
\hline 9 & Amaranthus viridis L. & Amaranthaceae & Herb & $\mathrm{Th}$ & $\mathrm{N}$ & 1.02 \\
\hline 10 & Aristida cyanantha Steud & Poaceae & Herb & $\mathrm{G}$ & $\mathrm{L}$ & 2.4 \\
\hline 11 & Artemisia scoparia Waldst. \& Kit & Asteraceae & Herb & $\mathrm{H}$ & $\mathrm{N}$ & 1.29 \\
\hline 12 & Arundo donax L. & Poaceae & Herb & G & $\mathrm{Mi}$ & 6.05 \\
\hline 13 & Asparagus adscendens Roxb. & Asparagaceae & Herb & $\mathrm{H}$ & $\mathrm{L}$ & 0.68 \\
\hline 14 & Avena sativa $\mathrm{L}$. & Poaceae & Herb & G & $\mathrm{L}$ & 0.84 \\
\hline 15 & Azadirachta indica A.Juss & Meliaceae & Tree & $\mathrm{Mp}$ & $\mathrm{Mi}$ & 0.39 \\
\hline 16 & Bauhinia variegata $\mathrm{L}$. & Fabaceae & Tree & $\mathrm{Mp}$ & $\mathrm{Me}$ & 0.53 \\
\hline 17 & Berberis lycium Royle & Berberidaceae & Shrub & $\mathrm{Np}$ & $\mathrm{Mi}$ & 0.51 \\
\hline 18 & Bidens pilosa $\mathrm{L}$. & Asteraceae & Herb & $\mathrm{Th}$ & $\mathrm{Mi}$ & 6.68 \\
\hline 19 & Bidens tripartita $\mathrm{L}$. & Asteraceae & Herb & $\mathrm{Th}$ & $\mathrm{Mi}$ & 0.21 \\
\hline 20 & Broussonetia papyrifera (L.) L Her.ex Vent & Moraceae & Tree & $\mathrm{Mp}$ & $\mathrm{Me}$ & 7.28 \\
\hline 21 & Bupleurum falcatum $\mathrm{L}$. & Apiaceae & Herb & $\mathrm{H}$ & $\mathrm{N}$ & 1.03 \\
\hline 22 & Callicarpa macrophylla Vahl & Verbenaceae & Shrub & $\mathrm{Np}$ & $\mathrm{Me}$ & 1.61 \\
\hline 23 & Calotropis gigantea $\mathrm{L}$. & Apocynaceae & Shrub & $\mathrm{Np}$ & $\mathrm{Mi}$ & 0.43 \\
\hline 24 & Cannabis sativa $\mathrm{L}$. & Cannabaceae & Herb & $\mathrm{Th}$ & $\mathrm{Mi}$ & 7.78 \\
\hline 25 & Capsella-bursa-pastoris (L.) Medik. & Brassicaceae & Herb & $\mathrm{Th}$ & $\mathrm{L}$ & 1.36 \\
\hline 26 & Carissa opaca Stapf ex Haines & Apocynaceae & Shrub & $\mathrm{Np}$ & $\mathrm{Mi}$ & 3.33 \\
\hline 27 & Celtis eriocarpa Decne. & Ulmaceae & Tree & $\mathrm{Mp}$ & $\mathrm{Mi}$ & 0.97 \\
\hline 28 & Chenopodium album $\mathrm{L}$. & Chenopodiaceae & Herb & $\mathrm{Th}$ & $\mathrm{Mi}$ & 3.75 \\
\hline 29 & Cichorium intybus L. & Asteraceae & Herb & $\mathrm{H}$ & $\mathrm{Mi}$ & 0.76 \\
\hline 30 & Cirsium ravens (L.) Scop. & Asteraceae & Herb & $\mathrm{H}$ & $\mathrm{Mi}$ & 0.89 \\
\hline 31 & Commelina benghalensis L. & Commelinaceae & Herb & $\mathrm{Th}$ & $\mathrm{Mi}$ & 0.32 \\
\hline 32 & Conyza bonariensis (L.) Cronquist & Asteraceae & Herb & $\mathrm{H}$ & $\mathrm{Mi}$ & 6.09 \\
\hline 33 & Cotinus coggyria L. & Anacardiaceae & Shrub & $\mathrm{Np}$ & $\mathrm{Mi}$ & 4.06 \\
\hline 34 & Cuscuta reflexa Roxb. & Cuscutaceae & Climber & $\mathrm{L}$ & $\mathrm{L}$ & 0.93 \\
\hline 35 & Cynodon dactylon L. Pers & Poaceae & Herb & G & $\mathrm{L}$ & 9.56 \\
\hline 36 & Cynoglossum lanceolatum Forssk. & Boraginaceae & Herb & $\mathrm{H}$ & $\mathrm{N}$ & 4.24 \\
\hline 37 & Cyperus exaltatus Retz. & Cyperaceae & Herb & G & $\mathrm{L}$ & 1.59 \\
\hline 38 & Cyperus rotundus $\mathrm{L}$. & Cyperaceae & Herb & G & $\mathrm{L}$ & 3.4 \\
\hline 39 & Dalbergia sissoo DC. & Papilionaceae & Tree & $\mathrm{Mp}$ & $\mathrm{Me}$ & 4.56 \\
\hline 40 & Debregeasia salicifolia (D Don) Rendle & Urticaceae & Shrub & $\mathrm{Np}$ & $\mathrm{Mi}$ & 2.45 \\
\hline 41 & Desmodium elegans DC. & Leguminosae & Shrub & $\mathrm{Np}$ & $\mathrm{Mi}$ & 0.63 \\
\hline
\end{tabular}


Table 1. Contimued.

\begin{tabular}{|c|c|c|c|c|c|c|}
\hline 42 & Dichanthium annulatum (Forssk.) Stapf. & Poaceae & Herb & G & $\mathrm{L}$ & 2 \\
\hline 43 & Diospyros lotus L. & Ebenaceae & Tree & $\mathrm{Mp}$ & $\mathrm{Me}$ & 0.42 \\
\hline 44 & Dodonaea viscosa (L.) Jacq. & Sapindaceae & Shrub & $\mathrm{Np}$ & $\mathrm{Mi}$ & 10.47 \\
\hline 45 & Duchesnea indica (Jacks.) Focke. & Rosaceae & Herb & Th & $\mathrm{Mi}$ & 1.32 \\
\hline 46 & Echinochloa colona (L.) Link & Poaceae & Herb & G & $\mathrm{L}$ & 1.03 \\
\hline 47 & Elaeagnus umbellata Thunb. & Elaeagnaceae & Shrub & $\mathrm{Np}$ & Mi & 1.32 \\
\hline 48 & Eleocharis indica (Lour) Druces & Cyperaceae & Herb & G & $\mathrm{L}$ & 2.69 \\
\hline 49 & Equisetum debile Roxb.ex Vaucher & Equisetaceae & Herb & G & $\mathrm{L}$ & 0.31 \\
\hline 50 & Erioscirpus comosus (Wall). & Cyperaceae & Herb & Th & $\mathrm{L}$ & 0.55 \\
\hline 51 & Nasturtium officinale $\mathrm{R}$. Br. & Brassicaceae & Herb & $\mathrm{H}$ & $\mathrm{N}$ & 0.49 \\
\hline 52 & Eucalyptus alba Reinw. ex Blume & Myrtaceae & Tree & $\mathrm{Mp}$ & $\mathrm{Mi}$ & 0.53 \\
\hline 53 & Euphorbia helioscopia L. & Euphorbiaceae & Herb & $\mathrm{Th}$ & Mi & 0.51 \\
\hline 54 & Euphorbia hirta L. & Euphorbiaceae & Herb & $\mathrm{Th}$ & $\mathrm{N}$ & 2.15 \\
\hline 55 & Euphorbia prostrata Aiton. & Euphorbiaceae & Herb & $\mathrm{Th}$ & $\mathrm{N}$ & 3.96 \\
\hline 56 & Ficus palmata Forssk. & Moraceae & Tree & $\mathrm{Mp}$ & $\mathrm{Me}$ & 2.56 \\
\hline 57 & Grevillea robusta A.Cunn.ex R.Br. & Proteaceae & Shrub & $\mathrm{Mp}$ & $\mathrm{Mi}$ & 0.58 \\
\hline 58 & Hibiscus rosa sinensis L. & Malvaceae & Shrub & $\mathrm{Np}$ & $\mathrm{Mi}$ & 0.85 \\
\hline 59 & Indigofera heterantha Brandis & Papilionaceae & Shrub & $\mathrm{Np}$ & $\mathrm{L}$ & 1.41 \\
\hline 60 & Ipomoaea lobata L. & Convolvulaceae & Climber & $\mathrm{L}$ & $\mathrm{Mi}$ & 0.44 \\
\hline 61 & Ipomoea eriocarpa $\mathrm{R} . \mathrm{Br}$. & Convolvulaceae & Climber & $\mathrm{L}$ & $\mathrm{Mi}$ & 0.07 \\
\hline 62 & Isodon rugosus (Wall .ex Benth) Codd & Lamiaceae & Shrub & $\mathrm{Np}$ & $\mathrm{Mi}$ & 0.35 \\
\hline 63 & Jasminum officinale L. & Oleaceae & Shrub & $\mathrm{Np}$ & $\mathrm{Mi}$ & 0.43 \\
\hline 64 & Juglans regia $\mathrm{L}$. & Juglandaceae & Tree & $\mathrm{Mp}$ & $\mathrm{Me}$ & 0.63 \\
\hline 65 & Justicia adhatoda $\mathrm{L}$ & Acanthaceae & Shrub & $\mathrm{Np}$ & Mi & 14.42 \\
\hline 66 & Lantana camara $\mathrm{L}$. & Verbenaceae & Shrub & $\mathrm{Np}$ & Mi. & 10.59 \\
\hline 67 & Lepidium bidentatum Montin. & Brassicaceae & Herb & $\mathrm{H}$ & Mi & 0.72 \\
\hline 68 & Mallotus philippensis (Lam.) Mull.Arg. & Euphorbiaceae & Tree & $\mathrm{Mp}$ & $\mathrm{Me}$ & 3.98 \\
\hline 69 & Malvastrum corommandelianum (L.) Gracke & Malvaceae & Herb & $\mathrm{H}$ & Mi & 3.1 \\
\hline 70 & Maytenus royleana (Wall ex Lawson) Cufod. & Celasteraceae & Shrub & $\mathrm{Np}$ & $\mathrm{N}$ & 1.88 \\
\hline 71 & Medicago sativa $\mathrm{L}$. & Papilionaceae & Herb & $\mathrm{H}$ & $\mathrm{N}$ & 0.55 \\
\hline 72 & Melia azedarach $\mathrm{L}$. & Meliaceae & Tree & $\mathrm{Mp}$ & $\mathrm{Me}$ & 3.94 \\
\hline 73 & Mentha royleana Benth. & Lamiaceae & Herb & $\mathrm{Th}$ & $\mathrm{Mi}$ & 2.5 \\
\hline 74 & Micromeria biflora (Buch Ham ex DDon) Benth. & Lamiaceae & Herb & Th & $\mathrm{L}$ & 1.1 \\
\hline 75 & Mirabilis jalapa $\mathrm{L}$. & Nyctaginaceae & Herb & Th & $\mathrm{Mi}$ & 0.68 \\
\hline 76 & Morus alba $\mathrm{L}$. & Moraceae & Tree & $\mathrm{Mp}$ & $\mathrm{Mi}$ & 1.74 \\
\hline 77 & Myrsine africana $\mathrm{L}$ & Myrsinaceae & Shrub & $\mathrm{Np}$ & $\mathrm{N}$ & 1.05 \\
\hline 78 & Nepeta erecta (Royle ex Benth) Benth & Lamiaceae & Herb & $\mathrm{H}$ & Mi & 3.02 \\
\hline 79 & Oenothera rosea L Her.exAiton & Onagraceae & Herb & $\mathrm{Th}$ & $\mathrm{Mi}$ & 3.37 \\
\hline 80 & Olea cuspidata Wall. Don & Oleaceae & Tree & $\mathrm{Mp}$ & $\mathrm{Mi}$ & 2.93 \\
\hline 81 & Otostegia limbata (Benth.)Boiss. & Lamiaceae & Shrub & $\mathrm{Np}$ & $\mathrm{Mi}$ & 1.95 \\
\hline 82 & Oxalis corniculata $\mathrm{L}$. & Oxalidaceae & Herb & $\mathrm{Th}$ & $\mathrm{N}$ & 5.98 \\
\hline 83 & Parthenium hysterophorus L. & Asteraceae & Herb & Th & Mi & 11.77 \\
\hline
\end{tabular}


Table 1. Contimued.

\begin{tabular}{|c|c|c|c|c|c|c|}
\hline 84 & Pennisetum unisetum (Nees) Benth. & Poaceae & Herb & G & $\mathrm{L}$ & 1.09 \\
\hline 85 & Persicaria barbata (L.) H.Hara & Polygonaceae & Herb & Th & $\mathrm{Mi}$ & 3.03 \\
\hline 86 & Phlomis tuberosa $\mathrm{L}$. & Lamiaceae & Herb & Th & $\mathrm{N}$ & 0.94 \\
\hline 87 & Phyllanthus fraternus G.L Webster. & Phyllanthaceae & Herb & Th & $\mathrm{N}$ & 1.71 \\
\hline 88 & Pinus roxburghii Sarg. & Pinaceae & Tree & $\mathrm{Mp}$ & $\mathrm{L}$ & 0.86 \\
\hline 89 & Pistacia integerimma J.L Stewart ex Brandis. & Anacardiaceae & Tree & $\mathrm{Mp}$ & $\mathrm{Me}$ & 0.4 \\
\hline 90 & Plantago lanceolata $\mathrm{L}$. & Plantaginaceae & Herb & $\mathrm{H}$ & $\mathrm{Mi}$ & 0.3 \\
\hline 91 & Plantago major L. & Plantaginaceae & Herb & $\mathrm{H}$ & $\mathrm{Mi}$ & 0.36 \\
\hline 92 & Populus alba L. & Salicaceae & Tree & $\mathrm{Mp}$ & $\mathrm{Mi}$ & 2.57 \\
\hline 93 & Prunus persica (L.) Batsch & Rosaceae & Tree & $\mathrm{Mp}$ & Mi & 0.2 \\
\hline 94 & Pteris cretica $\mathrm{L}$. & Pteridaceae & Fern & G & $\mathrm{Me}$ & 2.03 \\
\hline 95 & Pteris vittata $\mathrm{L}$. & Pteridaceae & Fern & G & $\mathrm{Me}$ & 0.21 \\
\hline 96 & Punica granatum $\mathrm{L}$. & Punicaceae & Shrub & $\mathrm{Np}$ & Mi & 5.96 \\
\hline 97 & Pyrus pashia Buch.Hem.ex D.Don. & Rosaceae & Tree & $\mathrm{Mp}$ & $\mathrm{Mi}$ & 1.08 \\
\hline 98 & Ricinus communis $\mathrm{L}$. & Euphorbiaceae & Shrub & $\mathrm{Np}$ & $\mathrm{Me}$ & 3.27 \\
\hline 99 & Rosa macrophylla Lindl. & Rosaceae & Shrub & $\mathrm{Np}$ & Mi & 1.75 \\
\hline 100 & Rubus ellipticus $\mathrm{Sm}$. & Rosaceae & Shrub & $\mathrm{Np}$ & $\mathrm{Mi}$ & 1.38 \\
\hline 101 & Rubus fruticosus G.N Jones. & Rosaceae & Shrub & $\mathrm{Np}$ & Mi & 4.51 \\
\hline 102 & Rumex hastatus D.Don. & Polygonaceae & Shrub & $\mathrm{Np}$ & $\mathrm{L}$ & 0.76 \\
\hline 103 & Rumex nepalensis Spreng. & Polygonaceae & Herb & Th & $\mathrm{Me}$ & 1.54 \\
\hline 104 & Saccharum spontaneum L. & Poaceae & Herb & G & $\mathrm{N}$ & 3.94 \\
\hline 105 & Salix alba L. & Salicaceae & Tree & $\mathrm{Mp}$ & Mi & 0.92 \\
\hline 106 & Setaria pumila (Poir.) Roem \& Schult. & Poaceae & Herb & G & $\mathrm{L}$ & 2.11 \\
\hline 107 & Solanum nigrum L. & Solanaceae & Herb & Th & Mi & 1.07 \\
\hline 108 & Solanum surattense Burm & Solanaceae & Herb & Th & Mi & 0.19 \\
\hline 109 & Sonchus asper (L.) Hill. & Asteraceae & Herb & Th & Mi & 2.01 \\
\hline 110 & Sorghum halepense (L.) Pers. & Poaceae & Herb & G & $\mathrm{L}$ & 3.11 \\
\hline 111 & Stellaria media (L.) Vill. & Caryophyllaceae & Herb & Th & $\mathrm{N}$ & 1.12 \\
\hline 112 & Tagetes minuta $\mathrm{L}$. & Asteraceae & Herb & Th & Lp & 2.74 \\
\hline 113 & Taraxacum officinale (L.) Weber ex FH Wigg. & Asteraceae & Herb & Th & Mi & 2.69 \\
\hline 114 & Trifolium repens $\mathrm{L}$. & Papilionaceae & Herb & $\mathrm{H}$ & $\mathrm{N}$ & 0.55 \\
\hline 115 & Verbascum thapsus L. & Scrophulariaceae & Herb & $\mathrm{Th}$ & $\mathrm{Me}$ & 2.36 \\
\hline 116 & Verbena tenuisecta Briq. & Verbenaceae & Herb & $\mathrm{Ch}$ & $\mathrm{Lp}$ & 3.62 \\
\hline 117 & Vitex negundo L. & Verbenaceae & Herb & $\mathrm{Np}$ & $\mathrm{N}$ & 1.32 \\
\hline 118 & Woodfordia fruticosa (L.) Kurz & Lythraceae & Shrub & $\mathrm{Np}$ & Mi & 2.59 \\
\hline 119 & Xanthium strumarium L. & Asteraceae & Herb & $\mathrm{H}$ & $\mathrm{Me}$ & 9.92 \\
\hline 120 & Zanthoxylum armatum DC & Rutaceae & Shrub & $\mathrm{Np}$ & Mi & 2.95 \\
\hline 121 & Ziziphus jujuba Mill. & Rhamnaceae & Shrub & $\mathrm{Np}$ & Mi & 2.05 \\
\hline
\end{tabular}

Key:

Life Form:

$\mathrm{Mp}=$ Megaphanerophytes; $\mathrm{Np}=$ Nanophanerophytes; $\mathrm{H}=$ Hemiryptophytes; $\mathrm{C}=$ Cryptophytes; $\mathrm{Th}=$ Therophytes;

$\mathrm{Ch}=$ Chameophytes; $\mathrm{G}=$ Geophytes

Leaf Spectra:

$\mathrm{Mi}=$ Mirophyll; $\mathrm{Me}=$ Meophyll $; \mathrm{N}=$ Nanophyll; $\mathrm{L}=$ Leptophyll 
Table 2. Invasion status and Phytogeographic origin of alien flora from Muzaffarabad

\begin{tabular}{|c|c|c|c|c|c|}
\hline Sr. no & Species name & Family & $\begin{array}{c}\text { Invasion } \\
\text { status }\end{array}$ & Origin & Reference \\
\hline 1 & Achyranthes aspera & Amaranthaceae & $\mathrm{Cn}$ & NAM & Kaul, (1986); Khuroo et al., 2007 \\
\hline 2 & Ailanthus altissima & Simaroubaceae & In & AS & Ara et al., (1995) \\
\hline 3 & Amaranthus spinosus & Amaranthaceae & In & SAM & Stewart, (1972); Khuroo et al., 2010 \\
\hline 4 & Artemisia scoparia & Asteraceae & $\mathrm{Nt}$ & AS,EU & Kaul, (1986); Khuroo et al., 2007 \\
\hline 5 & Arundo donax & Poaceae & Cs & $\mathrm{AF} ; \mathrm{EU}$ & Stewart, (1972) \\
\hline 6 & Bidens tripartita & Asteraceae & $\mathrm{Cn}$ & $\mathrm{AF} ; \mathrm{EU}$ & Kaul, (1986); Khuroo et al., 2010 \\
\hline 7 & Broussonetia papyrifera & Moraceae & In & AS & Stewart, (1972) \\
\hline 8 & Cannabis sativa & Cannabaceae & In & AS & Kaul, (1986) \\
\hline 9 & Capsella bursa-pastoris & Brassicaceae & In & EU & Kaul, (1986); Khuroo et al., 2007 \\
\hline 10 & Chenopodium album & Chenopodiaceae & In & EU & Kaul, (1986) \\
\hline 11 & Cichorium intybus & Asteraceae & In & EU & Kaul, (1986) ; Khuroo et al., 2010 \\
\hline 12 & Cirsium arvense & Asteraceae & In & AS & Stewart, (1972 \\
\hline 13 & Conyza bonariensis & Asteraceae & Cs & SAM & Kaul, (1986) ; Khuroo et al., 2010 \\
\hline 14 & Cyperus rotundus. & Cyperaceae & In & EU & Kaul, (1986); Khuroo et al., 2007 \\
\hline 15 & Elaeagnus umbellata. & Elaeagnaceae & $\mathrm{Nt}$ & AS & Singh and Kachroo, (1976) \\
\hline 16 & Euphorbia helioscopia & Euphorbiaceae & In & AS; EU & Kaul, (1986) \\
\hline 17 & Hibiscus rosa-sinensis & Malvaceae & $\mathrm{Nt}$ & AS & Naqshi et al., (1988) \\
\hline 18 & Ipomoea eriocarpa & Convolvulaceae & $\mathrm{Cn}$ & $\mathrm{AS} ; \mathrm{AU}$ & Kaul, (1986); Khuroo et al., 2010 \\
\hline 19 & Lantana camara. & Verbenaceaec & Cs & SAM & Stewart, (1972) \\
\hline 20 & Medicago sativa & Fabaceae & $\mathrm{Nt}$ & $\mathrm{AF} ; \mathrm{EU}$ & Dar et al., (2002) \\
\hline 21 & Melia azedarach & Meliaceae & $\mathrm{Cl}$ & AS & Ara et al., (1995) \\
\hline 22 & Mirabilis jalapa. & Nyctaginaceae & Cs & SAM & Stewart, (1972) \\
\hline 23 & Morus alba & Moraceae & $\mathrm{Nt}$ & AS & Dar et al., (2002) \\
\hline 24 & Nasturtium officinale. & Brassicaceae & $\mathrm{Nt}$ & EU & Kaul, (1986) \\
\hline 25 & Oenothera rosea & Onagraceae & In & SAM & Kaul, (1986); Khuroo et al., 2007 \\
\hline 26 & Oxalis corniculata & Oxalidaceae & $\mathrm{Nt}$ & $\mathrm{AS} ; \mathrm{EU}$ & Kaul, (1986); Khuroo et al., 2010 \\
\hline 27 & Parthenium hysterophorus & Asteraceae & Cs & SAM & Yaqoob et al., (1988) \\
\hline 28 & Plantago lanceolata. & Plantaginaceae & In & $\mathrm{AF} ; \mathrm{EU}$ & Kaul, (1986); Khuroo et al., 2010 \\
\hline 29 & Plantago major & Plantaginaceae & In & EU & Kaul, (1986); Khuroo et al., 2007 \\
\hline 30 & Populus alba & Salicaceae & $\mathrm{Nt}$ & EU & Javeid, (1972); Khuroo et al., 2010 \\
\hline 31 & Prunus persica & Rosaceae & $\mathrm{Cl}$ & AS & Dar et al., (2002) \\
\hline 32 & Ricinus communis. & Euphorbiaceae & Cs & $\mathrm{AF}$ & Stewart, (1972); Khuroo et al., 2007 \\
\hline 33 & Rumex hastatus & Polygonaceae & In & AS & Reshi, (1984); Khuroo et al., 2010 \\
\hline 34 & Rumex nepalensis & Polygonaceae & $\mathrm{Nt}$ & AF; EU & Munshi and Javied,(1986) \\
\hline 35 & Salix alba & Salicaceae & $\mathrm{Nt}$ & AS; AF; EU & Javeid, (1972); Khuroo et al., 2007 \\
\hline 36 & Setaria pumila & Poaceae & $\mathrm{Cn}$ & $\mathrm{AS} ; \mathrm{AF}$ & Stewart, (1972) \\
\hline 37 & Sorghum halepense. & Poaceae & In & EU & Kaul, (1986) ; Khuroo et al., 2010 \\
\hline 38 & Stellaria media. & Caryophyllacaeae & In & EU & Kaul, (1986) ; Khuroo et al., 2010 \\
\hline 39 & Tagetes minuta & Asteraceae & $\mathrm{Nt}$ & SAM & Singh and Kachroo, (1994) \\
\hline
\end{tabular}


Table 2. Continued.

\begin{tabular}{|c|c|c|c|c|c|}
\hline 40 & Taraxacum officinale & Asteraceae & In & EU & Kaul, (1986) \\
\hline 41 & Trifolium repens & Fabaceae & In & EU & Kaul, (1986); Khuroo et al., 2007 \\
\hline 42 & Verbascum thapsus & Scrophulariaceae & In & EU & Kaul, (1986); Khuroo et al., 2010 \\
\hline 43 & Xanthium strumarium. & Asteraceae & In & AF & Kaul, (1986); Khuroo et al., 2007 \\
\hline
\end{tabular}

Key

Origin: NAM: North America; SAM: South America; AF: Africa; EU: Europe; AU: Australia;

AS: Asia (Excluding the sub-continent).

Invasion status: Invasive aliens; Cl: Cultivated un-escaped aliens; Cs: Casual aliens; $\mathrm{Cn}$ : Casual or naturalized aliens;

Nt: Naturalized aliens; In:

(Categories used following Richardson et al., 2000; and Pysek et al., 2004).

Casual aliens were represented by $6(14 \%)$, whereas casual naturalized were represented by 4 species $(9 \%)$. Cultivated un-escaped aliens were represented by 2 species (5\%) (Table 3).

\section{Discussion}

Plant invasions are visibly a strong force of change, operating on a global scale and affecting many dimensions of society correlated with the increased trade and provision of ecosystem service. The present study was conducted to investigate the diversity and distribution of the invasive alien species and their potential impacts on vegetation of Muzaffarabad.

\section{Mode of Introduction of IAS in the Area}

The analysis of the invasion history and mode of investigation revealed that Lantana camara, Mirabilis jalapa and Ipomea eriocarpa have been introduced from different regions for use as ornamental plants $[13,21]$. Rumex hastatus and Artemisia scoparia are reported to have been introduced for medicinal purposes [22]. Ailanthus altissima, Elaeagnus umbellata, Melia azedarach, Broussonetia papyrifera, Populus alba and Salix alba are among the plants that have been introduced for plantation and revegetation of the barren land due to their high growth rates. Prunus persica and Morus alba have been introduced for horticulture due to their edible fruits. Chenopodium album, Ricinus communis and Rumex nepalensis plants have been introduced for food purposes and are used as vegetables [13, 23, 24]. Medicago sativa, Trifolium repens, Setaria pumila and Sorghum halepense have been introduced for utilization as fodder [25]. Hibiscus rosa-sinensis has been introduced for landscaping due to its aesthetic beauty. The highest number of the invasive alien plants was recorded to be introduced unintentionally. Amaranthus spinosus, Achyranthes aspera, Cichorium

Table 3. Quantitative phytosociological attributes of the investigated vegetation communities

\begin{tabular}{|c|c|c|c|c|c|c|c|c|c|c|}
\hline Site & Name & $\begin{array}{c}\text { Latitude } \\
\text { Longitude }\end{array}$ & $\begin{array}{c}\text { Altitude } \\
(\mathrm{m})\end{array}$ & $\begin{array}{c}\text { No of } \\
\text { species }\end{array}$ & $\begin{array}{c}\text { No of } \\
\text { IAS }\end{array}$ & $\begin{array}{c}\text { Simpsons } \\
\text { diversity }\end{array}$ & $\begin{array}{c}\text { Shannon } \\
\text { diversity }\end{array}$ & Richness & Evenness & $\begin{array}{c}\text { Maturity } \\
\text { index }\end{array}$ \\
\hline 1 & Langarpora & $\begin{array}{c}34.19 .035 \mathrm{~N} \\
73.31 .784 \mathrm{E}\end{array}$ & 731 & 43 & 17 & 0.96 & 2.57 & 1.62 & 0.68 & 30.69 \\
\hline 2 & Garidupata & $\begin{array}{l}34.15 .867 \mathrm{~N} \\
73.34 .650 \mathrm{E}\end{array}$ & 1199 & 50 & 21 & 0.98 & 2.81 & 2.11 & 0.71 & 27.4 \\
\hline 3 & Ambor & $\begin{array}{l}34.19 .214 \mathrm{~N} \\
73.28 .483 \mathrm{E}\end{array}$ & 646 & 48 & 23 & 0.97 & 2.40 & 2.37 & 0.62 & 26.1 \\
\hline 4 & Dulai & $\begin{array}{l}34.18 .279 \mathrm{~N} \\
73.28 .890 \mathrm{E}\end{array}$ & 659 & 43 & 18 & 0.97 & 2.76 & 2.36 & 0.73 & 27.23 \\
\hline 5 & Naluchee & $\begin{array}{l}34.21 .005 \mathrm{~N} \\
73.27 .669 \mathrm{E}\end{array}$ & 728 & 52 & 20 & 0.96 & 2.78 & 2.10 & 0.68 & 38.46 \\
\hline 6 & Shawai & $\begin{array}{l}34.23 .090 \mathrm{~N} \\
73.27 .539 \mathrm{E}\end{array}$ & 731 & 52 & 19 & 0.97 & 2.78 & 2.04 & 0.70 & 36.53 \\
\hline 7 & Subri & $\begin{array}{l}34.19 .554 \mathrm{~N} \\
73.30 .635 \mathrm{E}\end{array}$ & 732 & 37 & 16 & 0.96 & 2.19 & 1.61 & 0.71 & 33.78 \\
\hline 8 & Subri & $\begin{array}{l}34.22 .561 \mathrm{~N} \\
73.29 .769 \mathrm{E}\end{array}$ & 740 & 22 & 7 & 0.91 & 2.63 & 1.20 & 0.70 & 41.81 \\
\hline 9 & Majoi & $\begin{array}{l}34.15 .579 \mathrm{~N} \\
73.39 .079 \mathrm{E}\end{array}$ & 751 & 47 & 16 & 0.97 & 2.61 & 2.51 & 0.92 & 31.91 \\
\hline
\end{tabular}


intybus, Cirsium arvense, Conyza bonariensis, Parthenium hysterophorus, Tagetes minuta, Taraxacum officinale, Xanthium strumarium, Capsella bursapastoris, Cannabis sativa, Cyperus rotundus, Euphorbia helioscopia, Oenothera rosea, Oxalis corniculata, Plantago lanceolata, Plantago major, Arundo donax, Verbascum Thapsus, Stellaria media, Bidens tripartite and Nasturtium officinale are species reported to have been introduced unintentionally [13, 26-29]. The introduction of invasive aliens without any well-known purpose unintentionally in a non-native range has also been emphasized [30, 31].

\section{Phytogeographic Origion of Local Floral Elements}

On the basis of the origin of the IAS species, a total of 5 different geographical regions were identified in the present study. $39 \%$ of the IAS species were revealed to have their origion from Europe, followed by Asia contributing $29 \%$ of species. The IAS of African orgion were $16 \%$, followed by South American elements (14\%), whereas $2 \%$ of species had North American origins $[32,33]$.

Principal component analysis revealed the speceies distribution pattern in correlation with the specific sites in the study area. The primary vegetation data was verified by the species spread along the PCA biplot axis, showing the segregation of the dominant IAS including Parthenium hysterophorus, Lantana camara, Xanthium strumarium, Ailanthus altissima, Cannabis sativa and Broussonetia papyrifera indicating their over all dominance at all the study sites. Arundo donax showed close affinity with Site 7, showing its habitat specificity due to landslide disturbance with low organic matter, and increased solar insolation at the western aspect (Fig. 2).
Disturbance is among the key factors responsible for the spread and dominance of IAS [34, 35]. The disturbance patch invasion model supports our hypothesis that dsiturbances favour invasion by modifying the microclimate and disrupting inter-specific competitive interactions [36, 37]. Landslides and road construction were identified as factors promoting the establishment and spread of invasive species in any area. Results show the dominance of IAS like Arundo donax, Xanthium strumarium and Sorghum halepense at landslide and road-cutting disturbed sites [38]. The results of the present study are also in conformity with the experimental model of decreasing diversity levels with increasing invasive cover, which is evident from the Lantana camara-doiminated comuntities having relatively lower species diversity $[39,40]$. However, the values of species evenness were high at the disturbed sites as landslides break canopy dominance, allowing the invasive aliens species to grow rapidly.

The climatic and edaphic similarities between the original and new habitats are very important factors for establishing alien species [41]. A humid subtropical type of climate in lower elevations of the study area with highly leached soils are alike to Latin American home of species such as Lantana camara and Parthenium hysterophorus, enabling them to invade and abundantly colonize suburban vegetation of Muzaffarabad [42].

\section{Potential Impacts of the Dominant IAS in the Area}

Parthenium hysterophorus was present thoughout the investigated communities with an outstanding ability to change the vegetative structure in a wide range of habitats because of its efficient biological activity, allelloppathic effects and adaptability to varying microenvironments $[14,43]$. Regular contact with the plant over a prolonged

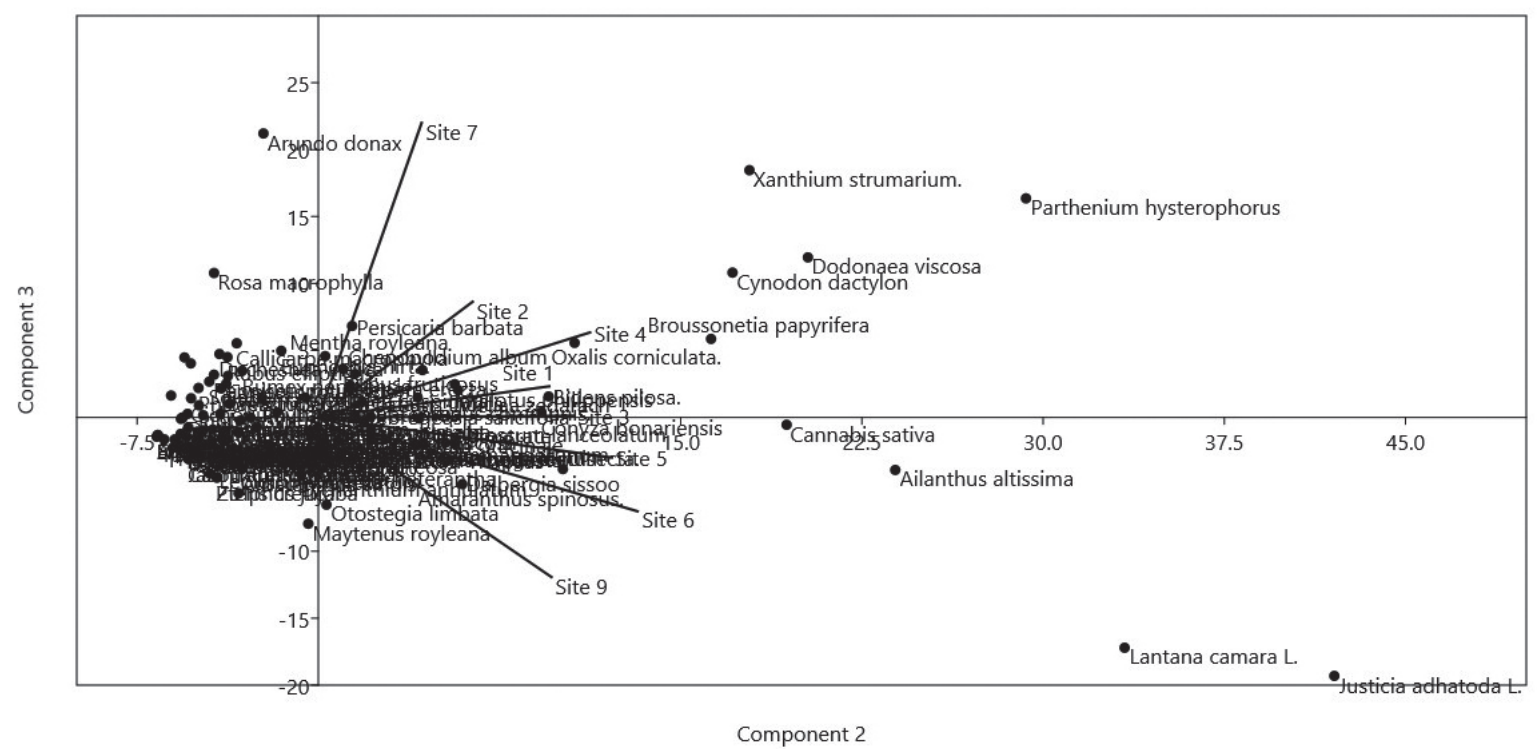

Fig 2. Principal Component Analysis Biplot oft he species data matrix. 
period is reported to produce allergenic asthma and dermatitis in humans. Lantana camara, a perennial shrub, was the second most abundant invasive attributed to the production of large number of seeds synchronized with an efficient dispersal mechanism facilitated by a variety of pollinators [33, 34]. Xanthium strumarium is a heat-loving and drought-resistant invasive plant reported from the area that prefers disturbed sites with open canopies with exposed soil surface. Exposue to this rough-textured plant with irritating surface hair causes itching and can lead to dermatitis and weeping eczema [45]. Ailanthus altissima and Melia azedarach were the abundantly distuributed deciduous invasive trees in the study area posing a serious threat to the native flora. These plants are highly competitive due to the secretion of allolopathic chemicals and altering the nutrient availability, thus inhibiting the growth of native plants $[11,44]$. Melia azedarach saplings also serve as a host for the ambrosia beetle (Xylosandrus mutilates), an insect that negatively impacts forest ecosystems. Arundo donax is a tall perennial shrub recorded from the disturbed sites that significantly modifies hydrological regimes and decreasing groundwater availability by transpiring large amounts of water, consuming three times the water of native plants. Arundo donax is also an extremely flammable plant, which increases the risk of accidental fires in suburban areas and poses a serious threat to people and property [14, 22]. Cichorium intybus is a perennial palatable herb recorded from the disturbed stands that prefereably inhabit wasteland, roadsides and sometimes cultivated land, and can survive dry, infertile conditions. Dairy products from Cichorium consuming livestock taste pungent, whereas its milky latex may cause dermatitis in humans. Cirsium arvense is another herbaceous perennial plant that produces allelopathic chemicals and displaces competing plant species [21, 32]. The spiny leaves are scratch and irritate, and are also highly flammable. Along with being a host for several parasites such as stalk borer, bean aphid and sod-web worm, Cirsium arvense is reported to collect nitrates, causing poisoning in livestock [43].

The spread of invasive alien species in the area is expected to further change the native community composition, reduce species diversity, retard ecosystem process, and cause ecological imbalance in the fragile Himalayan vegetation types [47, 48]. The spread of IAS in the urban area needs to be controlled by the application of integrated approaches involving urban landscape planning with the help of land cover maps, remote sensing tools and ecological modelling [49]. Immediate attention also needs to be given to the potential impacts of climate change on local climates and hydrology, paving the way for the introduction and spread of IAS in the area [50, 51].

Results emphasize the need for better management for early detection and reporting of invasive aliens. It is recommended to develop an integrated IAS detection network by establishing communication links between taxonomists, ecologists and land managers.

\section{Conflict of Interest}

The authors have not declared any conflict of interest.

\section{References}

1. GALLIEN L., CARBONI M. The community ecology of invasive species: where are we and what's next? Ecography, doi: 10.1111/ecog.02446. 2016.

2. PYS`EK P., JAROS`I'K V., HULME P.E., PERGL J, HEJDA M, SCHAFFNER U, VILA M. A global assessment of invasive plant impacts on resident species, communities and ecosystems: the interaction of impact measures, invading species' traits and environment. Glob Chang Biol 18, 1725, 2012.

3. IUCN/SSC Invasive Species Specialist Group (ISSG). Global Invasive Species Database version 2013-1. 2013.

4. VILA M., ESPINAR J.L., HEJDA M., HULME P.E., JAROSIK V., MARON J.L., PERGL J., SCHAFFNER U., SUN Y., PYSEK, P. Ecological impacts of invasive alien plants: a metaanalysis of their effects on species, communities and ecosystems. Ecol Lett 14, 702, 2011.

5. Global Invasive Alien Species Information Partnership (GIASIP). The GIASI Partnership Gateway. http:// giasipartner ship.myspecies.info. 2013

6. DENSLOW J.S. Managing dominance of invasive plants in wild lands. Curr Sci 93 (11), 1579, 2007.

7. CHARLES H, DUKES J.S. Impacts of Invasive Species on Ecosystem Services. Ecol Stud 193, 218, 2007.

8. DOSTA L.P, MU LLEROVA J., PYS`EK P., PERGL J., KLINEROVA T. The impact of an invasive plant changes over time. Ecol. Lett. 16, 1277, 2013.

9. GALLIEN L., MAZEL F., LAVERGNE S., RENAUD J., DOUZET R., THUILLER W. Contrasting the effects of environment, dispersal and biotic interactions to explain the distribution of invasive plants in alpine communities. Biol Invas 17, 1407, 2015.

10. CONCEPCION E.D., MORETTI M., ALTERMATT F., NOBIS M.P., OBRIST M.K. Impacts of urbanisation on biodiversity: the role of species mobility, degree of specialisation and spatial scale. Oikos 124, 1571, 2015.

11. HUSSAIN S.S., KHATOON S., MUHAMMAD R. Report on alien invasive species of Pakistan. Collaborative study of IUCN Pakistan, Cabi Rawalpindi and Botany Department, Karachi University, Karachi.n increased competitive ability. Front Ecol Environ 2, 436, 2000.

12. KHAN M.A., KHAN M.A., HUSSAIN M., MUJTABA G. Plant diversity and conservation status of Himalayan Region Poonch Valley Azad Kashmir. Pak J Pharm Sci 27 (5), 1215, 2014

13. CHANDRA SEKAR K. Invasive Alien Plants of Indian Himalayan Region - Diversity and Implication. Amer J Pl Sci, 3, 177, 2012.

14. CHANDRA S.K., PANDEY A., SRIVASTAVA S.K., GIRI L. Invasive alien plants of Himachal Pradesh, India, Ind Fores, 141 (5), 520, 2015.

15. GOAJK. Azad Jammu and Kashmir Statistical Book. Planning and Development Department Azad Government of the State of Jammu and Kashmir. 1, 2013.

16. PAK-MET. The normal of climatic data of Azaz Jammu and Kashmir Islamabad, Pakistan. Pakistan meterological department. 2014. 
17. DAUBENMIRE R.F. A canopy coverage method of vegetation analysis. Northwest Sci 33, 43, 1959.

18. AHMED M., SHAUKAT S.S. Status of vegetation analysis in Pakistan. Int J Biol Biotec 7 (3), 147, 2010.

19. PYŠEK P., RICHARDSON D.M., WILLIAMSON M. Predicting and explaining plant invasions through analysis of source area floras: some critical considerations. Diver Distrib 10, 179, 2004.

20. RICHARDSON D.M., PYSEK P., REJMANEK M., BARBOUR M.G., PANETTA F.D., WES C.J. Naturalization and invasion of alien plants concepts and definitions. Diver Distri 6, 93, 2000.

21. BHELlUM, B.L., MAGOTRA R.A. Catalogue of Flowering Plants of Doda, Kishtwar and Ramban Districts Kashmir Himalaya; Bishan Singh Mahenddra Pal Singh: Dehradun, India, 2012.

22. JARYAN V., UNIYAL S.K., GUPTA R.C., SINGH R.D. Alien flora of Indian Himalayan state of Himachal Pradesh. Env Monit Asses 185 (7), 6129, 2013.

23. KHUROO A.A., IRFAN R., ZAFAR R., DAR G.H., WAFAI B.A. The alien flora of Kashmir Himalaya, Biol Invasio, 9, 269, 2007.

24. KAUL M.K. Weed flora of Kashmir Valley. Scientific Publishers, Jodhpur, India. 1986.

25. STEWART R.R. Annotated catalogue of the vascular plants of West Pakistan and Kashmir. Fakhri Press, Karachi, Pakistan. 1972.

26. RESHI Z. Ecology and taxonomy of weeds of District Anantnag, Kashmir Himalaya. M. Phil Dissertation, Department of Botany, University of Kashmir, Srinagar, J\&K, India. 1984.

27. JAVED G.N. Salicaceae of Kashmir Himalaya. Ind Fores 98, 435, 1972.

28. ARA S., NAQSHI A.R. Ipomoea quamoclit Linn. (Convovulaceae) a tropical climber in temperate Kashmir. J Econ Taxon Bot 15, 471, 1991.

29. DAR G.H., BHAGAT R.C., KHAN M.A. Biodiversity of the Kashmir Himalaya. Valley Book House, Srinagar, India. 2002.

30. MUNSHI AH, JAVEID G.N. Systematic studies in Polygonaceae of Kashmir Himalaya. Scientifi Publishers, Jodhpur, India. 1986.

31. NAQSHI R.A., DAR G.H., JAVEID G.N., KACHROO P. Malvaceae of Jammu and Kashmir State, India. Ann Missouri Bot Garden 75, 1499, 1998.

32. Global Invasive Alien Species Information Partnership (GIASIP). The GIASIP Repository. http://giasip.gbif.org/ 2014.

33. SINGH K.P., SHUKLA A.N., SINGH J.S. State-level inventory of invasive alien plants, their source region and use potential. Curr Sci, 99 (1), 107, 2011.

34. IUCN/SSC Invasive Species Specialist Group (ISSG). Global Invasive Species Database version 2014-2. http://193.206.192.138/gisd. 2014.

35. STEVENS J.T., LATIMER A.M. Snowpack, fire, and forest disturbance: interactions affect montane invasions by non-native shrubs. Glob Change Biol 21, 2379, 2015.

36. ANDERSEN K.M., NAYLOR B.J., ENDRESS B.A., PARKS C.G. Contrasting distribution patterns of invasive and naturalized non-native species along environmental gradients in a semi-arid montane ecosystem. Appl Veg Sci 18, 683, 2015.
37. BENNETT A.E., THOMSEN M., STRAUSS S.Y. Multiple mechanisms enable invasive species to suppress native species. Amer J Bot 98, 1086, 2011.

38. DAVIDSON A.M., JENNIONS M., NICOTRA A.B. Do invasive species show higher phenotypic plasticity than native species and, if so, is it adaptive? A meta-analysis. Ecol Lett 14, 419, 2011.

39. TALUKDAR D. Allelopathic effects of Lantana camara. on Lathyrus sativus L.: Oxidative imbalance and cytogenetic consequences. J Allelop 31 (1), 71, 2013.

40. CHOYAL R., SHAR S.K. Allelopathic effects of Lantana camara on regeneration in Funaria hygrometrica. Ind $\mathrm{J}$ Fund and Appli Life Sci 1: 177, 2011.

41. MCDOUGALL K.L., ALEXANDER J.M., HAIDER S., PAUCHARD A., WALSH N.G., KUEFFER C. Alien flora of mountains: global comparisons for the development of local preventive measures against plant invasions. Divers Distrib 17, 103, 2011.

42. BHATIA S., CHOUDHARY R., KAUR B. Biology and effectiveness of the introduced biocontrol insect Zygogramma bicolorata (Coleoptera: Chrysomelidae) on the invasive weed Parthenium hysterophorus L. (Asteraceae) in Jammu (J\&K), India. Abstract in: $1^{\text {st }}$ Internation Workshop on Biocontrol of Parthenium hysterophorus, Kenya. 2010.

43. GHOSH D.K., MALLICK J.K. Flora of Darjeeling Himalayas and Foothills (Angiosperms); Bishen Singh Mahendra Pal Singh: Dehradun, India, 2014.

44. MURRELL C., GERBER E, KREBS C., PAREPA M., SCHAFFNER U., BOSSDORF O. Invasive knotweed affects native plants through allelopathy. Amer J Bot 98, 38, 2011.

45. GURLEY E.S., RAHMAN M., HOSSAIN M.J. Fatal Outbreak from Consuming Xanthium strumarium Seedlings during Time of Food Scarcity in Northeastern Bangladesh. PLOS ONE 5 (3), 97, 2010.

46. CABRA RIVAS I., SALDANA A., CASTRO-DIEZ P., GALLIEN, L. A multi-scale approach to identify invasion drivers and invaders' future dynamics. Biol Invas, 2, 411, 2015.

47. LEMBRECHTS O.J., ALEXANDER J.M., CAVIERES L.A., HAIDER S., LENOIR J., KUEFFER C., MCDOUGALL K., NAYLOR B.J., NUÑEZ M.A., PAUCHARD A., REW L.J., NIJS I., MILBAU A. Mountain roads shift native and non-native plant species' ranges. Ecography 40, 353, 2017.

48. PETITPIERRE B., MCDOUGALL K., SEIPEL T., BROENNIMANN O., GUISAN A., KUEFFER C. Will climate change increase the risk of plant invasions into mountains? Ecol Appl 26 (2), 530, 2016.

49. CETIN M., ZEREN I., SEVIK H., CAKIR C., AKPINAR H. A study on the determination of the natural park's sustainable tourism potential. Envir Monit Asses. 190 (3), 167, 2018.

50. CETIN M. Determination of bioclimatic comfort areas in landscape planning: A case study of Cide Coastline, Turk J Agri-Food Sci Tech 4 (9), 800, 2016.

51. CETIN M. Using GIS analysis to assess urban green space in terms of accessibility: case study in Kutahya. Int J Sust Dev Worl Ecol 22 (5), 420, 2015. 
